\title{
Individual- and area-level socioeconomic inequalities in diabetes mellitus in Saskatchewan between 2007 and 2012: a cross-sectional analysis
}

\author{
Daniel Fuller PhD, Joshua Neudorf MSc, Stuart Lockhart MPH, Charles Plante MA, Hazel Roberts MSc, \\ Thilina Bandara MPH, Cory Neudorf MD
}

\section{Abstract}

Background: Improving our understanding of social inequalities may improve prevention and treatment efforts for diabetes mellitus. We examined the association between individual- and area-level socioeconomic measures and physician-diagnosed diabetes in Saskatchewan over time.

Methods: In this cross-sectional study, we linked health administrative data with individual-level socioeconomic data from the Canadian Community Health Survey and area-level data from the 2006 Canadian census. We used general linear mixed-models regression to analyze the effect of each factor, controlling for geographic and demographic measures.

Results: Area-level deprivation was associated with medically diagnosed type 2 diabetes mellitus after adjustment for the individuallevel factors of age, sex, household income and education. Individuals residing in areas ranked in the least deprived quintile had a lower likelihood of diabetes than those in the most deprived quintile (odds ratio $0.40,95 \%$ confidence interval $0.18-0.88$ ). However, this disparity existed only in urban areas. This result may reflect less pronounced health inequalities in rural areas, greater socioeconomic heterogeneity, larger geographic units or some combination of these factors.

Interpretation: Individual- and area-level socioeconomic factors were associated with the likelihood of medically diagnosed diabetes; however, the strength of this association varied between urban and rural communities. Acknowledgement of area-level deprivation as a modifiable risk factor related to the prevalence of diabetes is important in the development of effective interventions for urban, but not rural, areas.

tudies examining the association between socioeconomic deprivation and diabetes mellitus have shown that inequalities exist at both individual and area levels. ${ }^{1-3} \mathrm{~A}$ systematic review and meta-analysis showed that individuals with low income had a relative risk of diabetes that was 1.4 times (95\% confidence interval [CI] 1.04-1.88) greater than that of individuals with high income. ${ }^{1}$ Studies in Canada have shown associations between area-level deprivation and diabetes. ${ }^{2}$ This work has led to proposals that addressing geographic variation in health services factors and socioeconomic deprivation is essential for population health improvements. ${ }^{3,4}$

One challenge that researchers confront in studying socioeconomic variation in health research is variation in geographic units between urban and rural areas. Past research has shown that area-level socioeconomic deprivation measures may be biased in rural areas because of the large land area and the heterogeneity of socioeconomic characteristics relative to the smaller urban land areas of equal population size. ${ }^{5,6}$ At the individual level, a deprivation index may not be appropriate for differentiating between urban and rural areas, and a single-item measure, such as income, may be more appropriate. ${ }^{6}$

Guided by the World Health Organization's Urban HEART (Health Equity Assessment and Response Tool), ${ }^{7}$ we developed local indicators, including area-level deprivation and individual-level income and education. For the current study, we examined the association between area-level socioeconomic deprivation and individual-level income and education and medically diagnosed diabetes in Saskatchewan between 2007

Competing interests: None declared.

This article has been peer reviewed.

Correspondence to: Cory Neudorf, cory.neudorf@usask.ca CMAJ Open 2019. DOI:10.9778/cmajo.20180042 
and 2012. We hypothesized that both area-level socioeconomic deprivation and individual-level income and education would be associated with diabetes over time, but that this effect would be modified by urban and rural contexts.

\section{Methods}

In this cross-sectional study, we examined individual medically diagnosed prevalent cases of diabetes in Saskatchewan using health administrative data linked to individual-level socioeconomic data from the Canadian Community Health Survey and linked again with area-level socioeconomic information derived from the 2006 Canadian census. ${ }^{8}$ These linkages were required because individual socioeconomic data are not available in health administrative data; however, health administrative data do contain patients' postal codes, which allowed us to link administrative data to area-level census geographic units.

We performed this linkage using a unique identifier assigned by the Government of Saskatchewan to all those eligible for health services. We linked these individuals to census dissemination areas using Statistics Canada's postal code conversion file, ${ }^{9}$ which allowed us to convert postal codes assigned to individual records to dissemination area-level variables derived from the 2006 census. Health administrative, ${ }^{10}$ Canadian Community Health Survey ${ }^{11}$ and postal code conversion file ${ }^{12}$ data sets are reliable, valid and commonly used for examining social inequalities in health.

\section{Definition of diabetes cases}

Our analysis was based on the yearly period prevalence of diabetes. We included in the analysis all cases of diabetes in patients older than 1 year of age in a given year. We defined a prevalent case of diabetes in a given year as a person with at least 1 hospital discharge record having a diagnosis of diabetes in any diagnostic field of the International Statistical Classification of Diseases and Related Health Problems, 10th revision (Canadian version) (E10, E11, E12 or E14) and/or 2 or more physician service claims within a 730 -day $(2-y r)$ period with a diabetes diagnosis code of 250 in the International Classification of Diseases, 9th revision. ${ }^{13} \mathrm{We}$ excluded cases of gestational diabetes, and counted multiple hospital or physician visits on the same day only once. We excluded transfers of the same patient between hospitals to avoid double counting.

\section{Area-level socioeconomic status}

The area-level unit of analysis was the dissemination area, which consists of 400-700 persons and is the smallest geographic unit available in census data. We operationalized arealevel socioeconomic status using the dissemination area-level deprivation index developed by Pampalon and colleagues. ${ }^{14}$ We obtained these data from the Institut national de santé publique. This index is made up of 2 dimensions: material and social deprivation. The material deprivation dimension incorporates the proportion of individuals age 15 years or older without a high school diploma, the employment-population ratio for people age 15 years or older, and the average income of those age 15 years or older. The social deprivation dimension incorporates the proportion of individuals age 15 years or older who live alone; the proportion of individuals age 15 years or older who are separated, divorced or widowed; and the proportion of single-parent families. We combined material and social deprivation scores to create quintiles of total deprivation for each dissemination area in Saskatchewan, as described by Pampalon and colleagues. ${ }^{14}$

\section{Individual-level socioeconomic status}

We controlled for education and household income. We operationalized education as the highest level of education achieved (according to variable EDUDR04 derived from the Canadian Community Health Survey), categorized as less than high school education, completion of high school and completion of at least some postsecondary education. Income was defined as total household income from all sources (the variable INCDHH derived from the Canadian Community Health Survey). We categorized income as \$0-\$19 999, $\$ 20$ 000-\$49 999, \$50 000-\$79 999, or \$80 000 or more. We applied these categorizations to represent education and income distribution while ensuring sufficient sample size in each category.

\section{Covariables}

We included as covariables age, sex and whether the individual lived in an urban or rural area. We categorized age as less than 35 years, 35-44 years, 45-59 years, 60-79 years, or 80 years or older; we recorded sex as self-reported in the Canadian Community Health Survey. We defined urban areas as dissemination areas having a population of 1000 or more and a population density of 400 or more per square kilometre, which is the definition used in the Canadian Community Health Survey. We also included dummy variables for years 2007 through 2011 to control for periodic variation.

\section{Statistical analysis}

We used multilevel logistic regression to estimate the prevalence of diabetes (yes/no), controlling for random effects at the level of dissemination area. We fitted models for the population of Saskatchewan as a whole, and fitted separate models for urban and rural areas. Multilevel models included bivariable screening for significance and a stepwise approach for multivariable model-building. First, we fit null models. Then, we included area-level factors. Finally, we included all individual socioeconomic variables, covariables and a categorical variable representing each year. We fitted all of our models using SAS general linear mixed-models with maximum likelihood subject-specific pseudo-likelihood estimation (SAS Institute Inc.). All reported results were weighted using the Canadian Community Health Survey weight variable (SAS analysis code available at https://github.com/walkabilly/ diabetes_inequality).

\section{Ethics approval}

The study was approved by the University of Saskatchewan Biomedical Research Ethics Board (Bio no. 12-268). 


\section{Results}

A total of 6331934 people were covered by Saskatchewan Health between 2007 and 2012, an average of 1055322 per year. This total included, for the same period, 457059 prevalent cases of diabetes. Across all years, data from the Canadian Community Health Survey included 41468 respondents who provided permission for data linkage. After exclusions, we linked Saskatchewan Health data for 15720 individuals, including 1498 prevalent cases of diabetes, across all years. We also linked these individuals to dissemination areas. Of the 2431 dissemination areas, 1018 were missing, as a result of our choice to use a single link indicator, which meant that postal codes overlapping multiple dissemination areas were assigned to only one; this left 1413 dissemination areas in the final data. Derivation of the study sample is shown in Figure 1.

Table 1 reports weighted and unweighted descriptive statistics for the variables used in our analysis. The following summary is presented in terms of weighted results. Across all years, the proportion of people with prevalent diabetes was $7.6 \%$. The youngest age group, less than 35 years old, represented $38.5 \%$ of the population and the oldest $(\geq 80 \mathrm{yr})$ $4.5 \%$. Males and females represented $49.4 \%$ and $50.6 \%$ of the population, respectively. The percentage of people with household income less than $\$ 20000$ was $21.4 \%$, and $54.1 \%$ had a household income of $\$ 50000$ or more per year. The percentage of those with less than high school education was $24.2 \%$, whereas $48.3 \%$ had some postsecondary education. About one-quarter (22.3\%) of the population resided in rural areas.

\section{Association between socioeconomic variables and diabetes mellitus}

Table 2 reports the results of the 3 logistic regression models that we fitted: for Saskatchewan as a whole, for urban areas only and for rural areas only. "Level 2" denotes variables

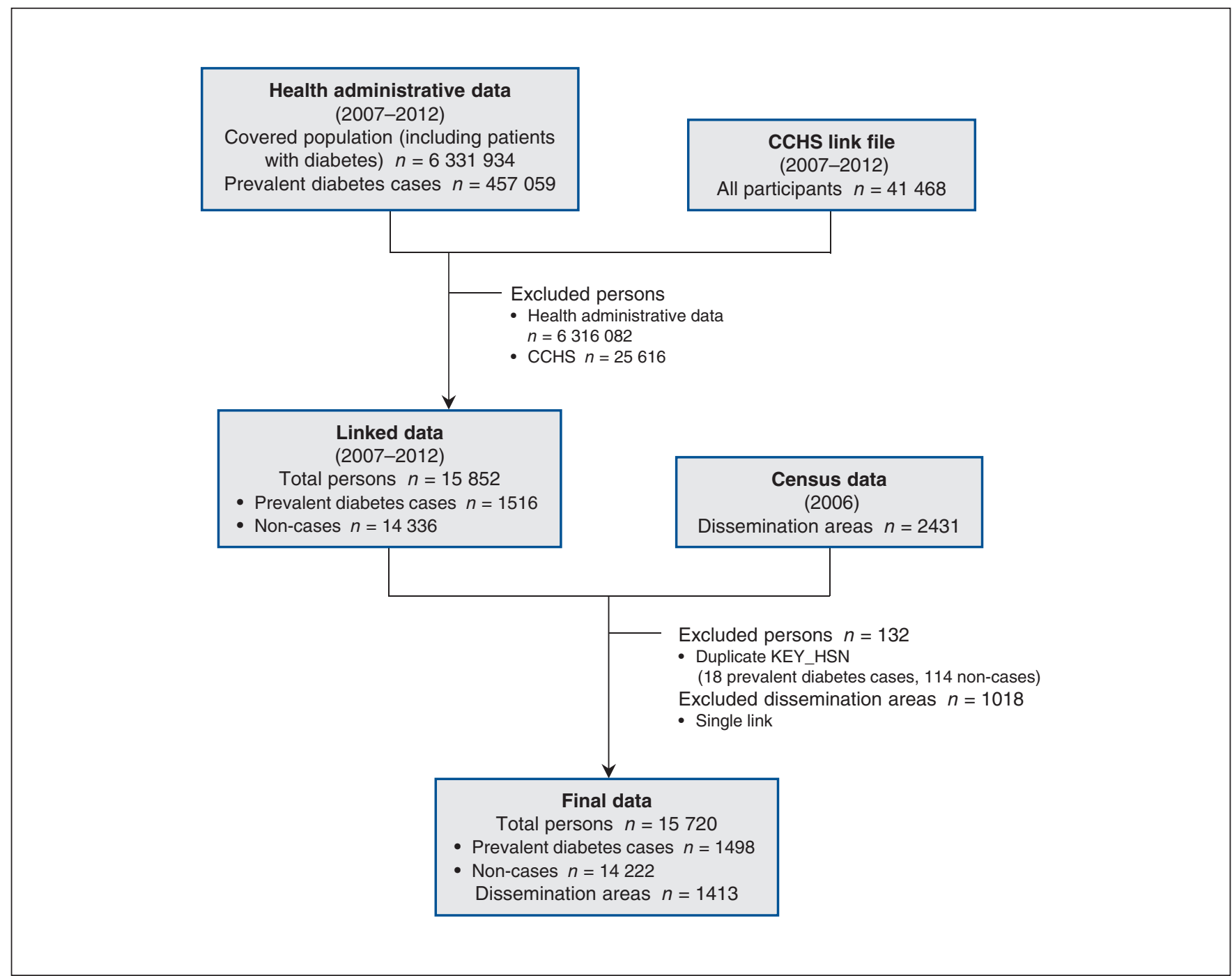

Figure 1: Flow chart for selection of diabetes mellitus cases from health administrative data, Canadian Community Health Survey (CCHS) link file data and census data, Saskatchewan, 2007-2012. Prevalent diabetes cases are included within the total covered population. "KEY_HSN" represents the linking variable for the data. 


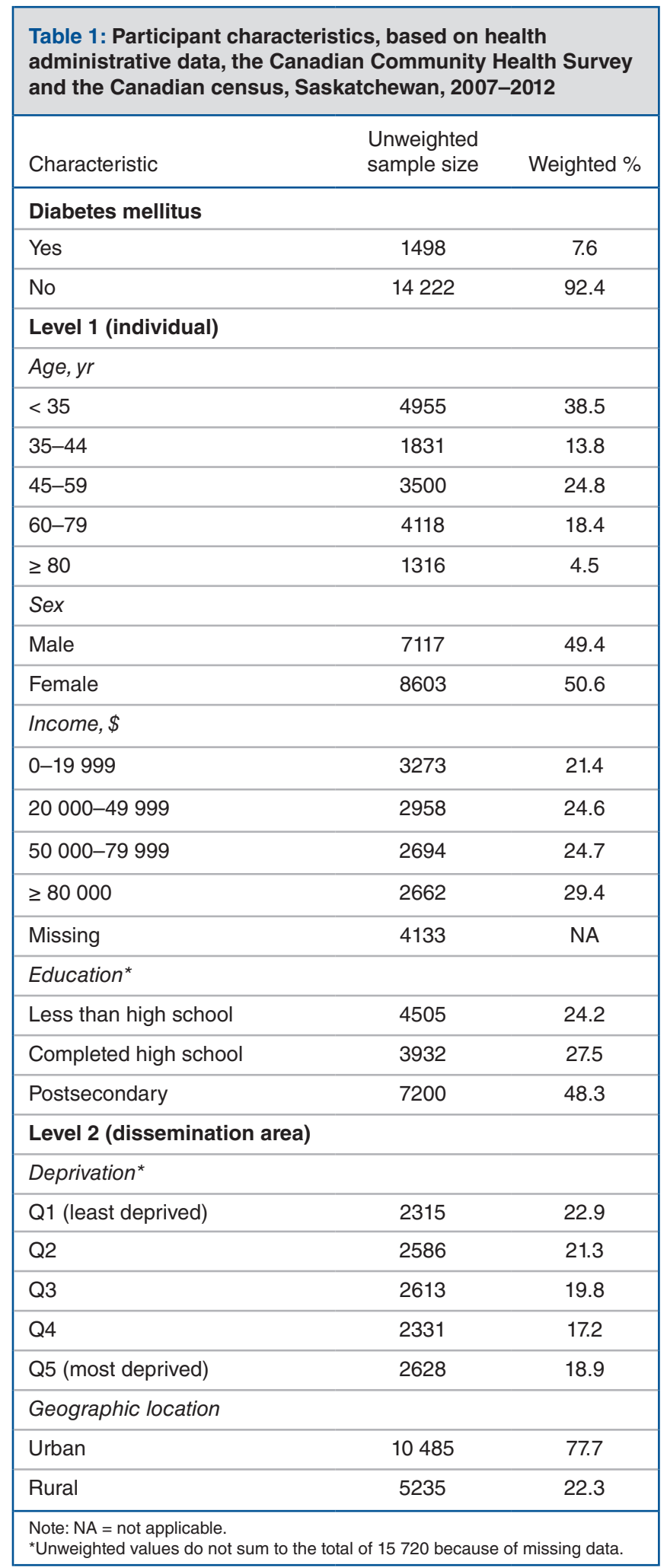

having effects at the level of dissemination area and "level 1" denotes variables having effects at the individual level.

In the model for Saskatchewan as a whole, people in urban areas had a lower likelihood of diabetes than those in rural areas (odds ratio [OR] 0.46 , 95\% CI 0.27-0.79). Relative to people in the most deprived quintile, those in the least deprived quintile had a lower likelihood of diabetes (OR 0.40, 95\% CI 0.18-0.88). There were no significant differences between quintiles 2, 3 and 4 and quintile 5, the most deprived quintile. Individual-level socioeconomic variables were associated with diabetes across the range of their distributions. Relative to people earning less than $\$ 20000$, those earning incomes of $\$ 20000$ to $\$ 49999$ (OR 0.64, 95\% CI 0.63-0.65), $\$ 50000$ to $\$ 79999$ (OR 0.65, 95\% CI 0.64-0.67) and $\$ 80000$ or more (OR 0.64, 95\% CI 0.63-0.66) all had a lower likelihood of diabetes. Relative to those with some postsecondary education, those with less than a high school education (OR 1.51, 95\% CI 1.49-1.54) and those who had completed high school (OR 1.08, 95\% CI 1.06-1.10) were more likely to have a diagnosis of diabetes.

\section{Urban and rural associations}

We found the same relations between socioeconomic status and period prevalence of diabetes for urban areas as for Saskatchewan as a whole, with those residing in the least deprived quintile having a lower likelihood of diabetes than those residing in the most deprived quintile (OR 0.32, 95\% CI $0.13-$ $0.78)$. Area-level deprivation was not associated with the likelihood of diabetes in rural areas.

We hypothesized that individual-level socioeconomic variables would be associated with diabetes in both urban and rural settings. We found that in both urban areas (OR $0.72,95 \%$ CI 0.70-0.73) and rural areas (OR 0.43, 95\% CI $0.41-0.45)$, those earning at least $\$ 80000$ per year were less likely to have a diagnosis of diabetes than those earning less than \$20 000 per year. There was an individual-level income gradient for diabetes in both rural areas. In urban areas, there were differences between the lowest income quintile and other quintiles. The gradient was small and not in the expected direction.

In both urban areas (OR 1.50, 95\% CI 1.47-1.53) and rural areas (OR 1.43, 95\% CI 1.39-1.48), those who had not completed high school were more likely than those with some postsecondary education to have a diagnosis of diabetes. However, unlike the situation for the province as a whole, those in rural settings who had completed their high school education were less likely to have a diagnosis of diabetes than those who had some postsecondary education (OR 0.87, 95\% CI 0.84-0.91).

\section{Interpretation}

The objective of this study was to examine associations between individual- and area-level socioeconomic deprivation and medically diagnosed diabetes in Saskatchewan between 2007 and 2012. As a secondary objective, we stratified the analyses by urban and rural location, to examine potential differences in these associations by region. We found that arealevel deprivation was associated with medically diagnosed diabetes mellitus after adjustment for individual-level factors in Saskatchewan overall and in urban areas. We did not detect area-level inequalities in diabetes in rural areas. 


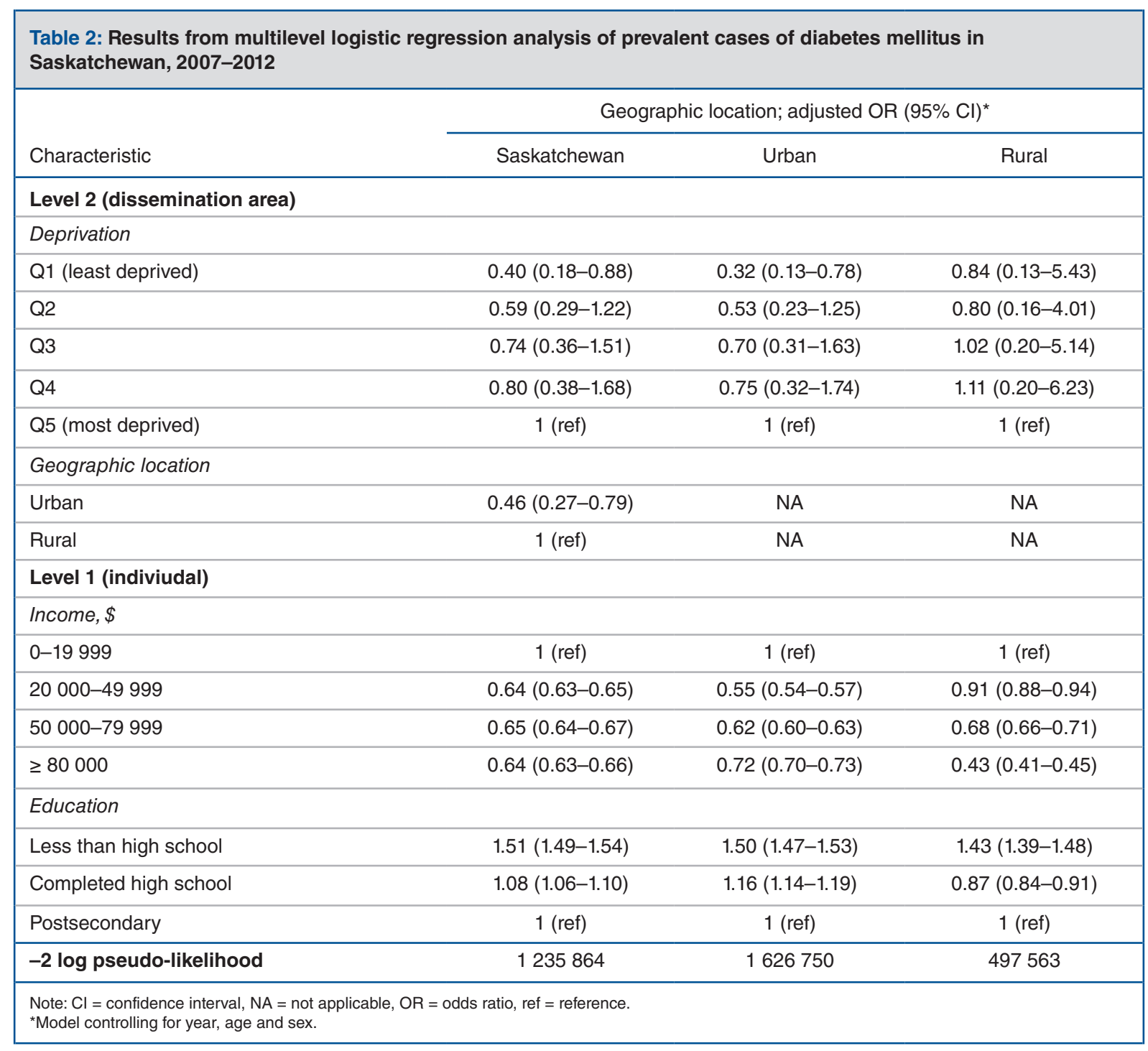

These results are consistent with past research showing that area-level socioeconomic deprivation is associated with a greater likelihood of diabetes, even when the model is controlled for individual-level socioeconomic characteristics. The estimated association between area-level socioeconomic deprivation and diabetes was higher than reported in previous studies. We found a 2.5 times greater likelihood of diabetes in socioeconomically disadvantaged areas, whereas 2 previous studies estimated effects of 1.8 and 1.4 times greater likelihood of diabetes, respectively. ${ }^{15,16}$ Area-level material deprivation is also predictive of the future risk of diabetes for females (2.4 times greater risk) and males (1.6 times greater risk). ${ }^{17}$

At the individual level, and in Saskatchewan as a whole, individual-level socioeconomic deprivation was associated with greater period prevalence of diabetes. People in household income categories of $\$ 20000$ or more had lower likelihood of diabetes than those whose household income was less than $\$ 20$ 000. In addition, people without any postsecondary education (whether or not they had completed high school) had a greater likelihood of diabetes than those with at least some postsecondary education.

In the model based solely on urban areas, all of the wealthier income groups fared better than the least well-off group, but the gradient was the opposite of what might have been expected. This finding may have been related to changes in household income and chronic disease over time, associated with changes in the population composition due to immigration to urban Saskatchewan over the study period. ${ }^{18,19}$

In rural areas, the likelihood of diabetes was lower in the group with completed high school education than in the group with postsecondary education. In Saskatchewan, less educated rural residents may work in physically demanding jobs in farming communities, which could explain this lower likelihood of diabetes. ${ }^{20}$

Our results suggest that caution should be exercised when interpreting results of studies about health inequalities in rural settings. Area-based measures of deprivation are less similar to individual-level measures of deprivation in rural areas. ${ }^{21}$ In rural settings, area units encompass considerably larger 
geographic areas and greater heterogeneity in socioeconomic status than in geographically smaller urban areas of equal population size. ${ }^{22}$ Multifactor deprivation indices may have too many factors to be effective in rural settings, and single-item, area-based measures may be preferable for rural settings. 5,23 Overall, and consistent with recent assessment of the practical utility of diabetes intervention research, more evidence is needed to inform and tailor preventive measures based on individual- and area-level socioeconomic factors. ${ }^{24}$

\section{Limitations}

This work had limitations related to the use of health administrative and census geography data, and to the lack of representation of Indigenous and First Nations populations. Because of limitations in data access, we did not present bivariable associations between covariables and the outcome. The use of health administrative data for research is common, and there is limited risk of bias if proper procedures are followed. ${ }^{25,26}$ In Saskatchewan, about 33\% of general practitioners and $38 \%$ of specialists "shadow bill" (i.e., submit billings despite receiving a salary and not being paid directly from their billings). These physicians typically do not shadow bill $100 \%$ of their work, but there is no auditing to determine the prevalence of this practice in Saskatchewan. ${ }^{27}$ Given the long period of the study, it is also possible that physician turnover and changes in diagnostic practices over time affected the results. ${ }^{28}$

We used deprivation data from the 2006 census. Our method assumed no change in area-level deprivation between 2007 and 2012 in Saskatchewan, which might have led to misclassification. As background for this study, we compared area-level deprivation between 2001 and 2006. Our results showed that $45 \%$ of dissemination areas did not change deprivation quintiles, and $37 \%$ of changes were within 1 deprivation quintile. There was also potential misclassification of postal codes to dissemination areas.

Our analysis did not include potentially relevant covariables such as demographic changes or migration patterns. Also, our analyses did not include on-reserve First Nations communities, because neither the Canadian Community Health Survey nor the census-based deprivation index include these communities. More than half of the Indigenous population of Saskatchewan lives off reserve; however, we could not identify this population because there is no measure of Indigenous or First Nations status in health administrative data for Saskatchewan. ${ }^{29}$

\section{Conclusion}

In this study, we examined individual- and area-level socioeconomic inequalities in diabetes in Saskatchewan from 2007 to 2012. Area-level socioeconomic deprivation was associated with a greater likelihood of diabetes, when we controlled for individual-level income and education. Our results further suggest that this relation was mediated by whether people resided in urban or rural areas and that area-level socioeconomic factors should especially be considered in strategies for diabetes prevention in urban settings.
Targeted measures designed to improve health equity represent a key instrument for improving population health. Our results suggest that the most effective approach is likely to differ between urban and rural settings. Place-based interventions are more likely to work in cities, where populations are densely distributed; however, targeting in rural areas should be guided by individual characteristics.

\section{References}

1. Agardh E, Allebeck P, Hallqvist J, et al. Type 2 diabetes incidence and socioeconomic position: a systematic review and meta-analysis. Int 7 Epidemiol 2011;40:804-18.

2. Lemstra M, Neudorf C, Opondo J. Health disparity by neighbourhood income. Can 7 Public Health 2006;97:435-9.

3. Gabb G, Arnolda L. Geographic location as a modifiable cardiac risk factor. CMA7 2017;189:E482-3.

4. Cox M, Boyle PJ, Davey PG, et al. Locality deprivation and type 2 diabetes incidence: a local test of relative inequalities. Soc Sci Med 2007;65:1953-64.

5. Terashima M, Rainham DGC, Levy AR. A small-area analysis of inequalities in chronic disease prevalence across urban and non-urban communities in the province of Nova Scotia, Canada, 2007-2011. BMF Open 2014;4:e004459.

6. Pampalon R, Martinez J, Hamel D. Does living in rural areas make a difference for health in Québec? Health Place 2006;12:421-35.

7. Urban HEART: urban health equity assessment and response tool: user manual. Geneva: World Health Organization; 2010.

8. 2006 census of population [census data sets]. Ottawa: Statistics Canada; modified 2018 Oct. 31. Available: www12.statcan.gc.ca/datasets/Index-eng.cfm (accessed 2018 Nov. 23).

9. Postal code ${ }^{O M}$ conversion file (PCCF), reference guide. Cat no. 92-154-G. Ottawa: Statistics Canada; 2017 Dec. 13. Available: www.canadapost.ca/cpc/assets/cpc/ uploads/files/marketing/2017-postal-code-conversion-file-reference-guide-en. pdf (accessed 2018 Aug. 2).

10. Health status reports. Saskatoon: Government of Saskatchewan; 2018. Available: www.saskatchewan.ca/government/government-structure/ministries/health/ other-reports/health-status-reports (accessed 2018 Nov. 23).

11. Canadian Community Health Survey - annual component (CCHS). Ottawa: Statistics Canada; modified 2018 Nov. 13. Available: www23.statcan.gc.ca/imdb/ p2SV.pl? Function=getSurvey\&SDDS=3226\#a3 (accessed 2018 Nov. 29).

12. 5. Data quality. In: Postal code ${ }^{O M}$ conversion file (PCCF), reference guide. Cat no. 92-154-G. Ottawa: Statistics Canada; modified 2015 Nov. 30. Available: www150. statcan.gc.ca/n1/pub/92-154-g/2013001/qual-eng.htm (accessed 2018 Nov. 23).

13. Report from the Canadian Chronic Disease Surveillance System: bypertension in Canada, 2010. Ottawa: Public Health Agency of Canada; 2010.

14. Pampalon R, Hamel D, Gamache P. A deprivation index for healthy planning in Canada. Chronic Dis Can 2009;29:178-91.

15. Rachele JN, Giles-Corti B, Turrell G. Neighbourhood disadvantage and selfreported type 2 diabetes, heart disease and comorbidity: a cross-sectional multilevel study. Ann Epidemiol 2016;26:146-50.

16. Grundmann N, Mielck A, Siegel M, et al. Area deprivation and the prevalence of type 2 diabetes and obesity: analysis at the municipality level in Germany. BMC Public Health 2014;14:1264.

17. Rivera LA, Lebenbaum M, Rosella LC. The influence of socioeconomic status on future risk for developing type 2 diabetes in the Canadian population between 2011 and 2022: differential associations by sex. Int 7 Equity Health $2015 ; 14: 101$.

18. Chiu M, Austin PC, Manuel DG, et al. Comparison of cardiovascular risk profiles among ethnic groups using population health surveys between 1996 and 2007. CMA7 2010;182:E301-10.

19. Quan H, Fong A, De Coster C, et al. Variation in health services utilization among ethnic populations. CMA7 2006;174:787-91.

20. Dyck R, Karunanayake C, Pahwa P, et al.; Saskatchewan Rural Health Study Group. Prevalence, risk factors and co-morbidities of diabetes among adults in rural Saskatchewan: the influence of farm residence and agriculture-related exposures. BMC Public Health 2013;13:7.

21. Barnett S, Roderick P, Martin D, et al. A multilevel analysis of the effects of rurality and social deprivation on premature limiting long-term illness. 7 Epidemiol Community Health 2001;55:44-51.

22. Schuurman N, Bell N, Dunn JR, et al. Deprivation indices, population health and geography: an evaluation of the spatial effectiveness of indices at multiple scales. F Urban Health 2007;84:591-603.

23. Mustard CA, Derksen S, Berthelot JM, et al. Assessing ecologic proxies for household income: a comparison of household and neighbourhood level income measures in the study of population health status. Health Place 1999;5:157-71.

24. Cefalu W'T, Buse JB, Tuomilehto J, et al. Update and next steps for real-world translation of interventions for type 2 diabetes prevention: reflections from a Diabetes Care Editors' Expert Forum. Diabetes Care 2016;39:1186-201. 
25. van Walraven C, Austin P. Administrative database research has unique characteristics that can risk biased results. 7 Clin Epidemiol 2012;65:126-31.

26. Virnig BA, McBean M. Administrative data for public health surveillance and planning. Annu Rev Public Health 2001;22:213-30.

27. Data quality documentation, Discharge Abstract Database - current-year information, 2012-2013. Ottawa: Canadian Institute for Health Information; 2013. Available: www.cihi.ca/sites/default/files/dad_data_quality_12_13_en_0.pdf (accessed 2018 Aug. 2).

28. Song Y, Skinner J, Bynum J, et al. Regional variations in diagnostic practices. NEngl 7 Med 2010;363:45-53.

29. Focus on geography series, 2016 census. Cat no 98-404-X2016001. Ottawa: Statistics Canada; 2017. Available: www12.statcan.gc.ca/census-recensement/2016/ as-sa/fogs-spg/Facts-pr-eng.cfm?LANG=Eng\& GK=PR\&GC=47\&TOPIC=9 (accessed 2018 Nov. 29).

Affiliations: School of Human Kinetics and Recreation (Fuller), Memorial University of Newfoundland, St. John's, Nfld.; Department of Community Health and Epidemiology (J. Neudorf, Plante, Roberts,
Bandara, C. Neudorf), University of Saskatchewan, Saskatoon, Sask.; School of Medicine (Lockhart), Trinity College, Dublin, Ireland

Contributors: Daniel Fuller, Stuart Lockhart and Cory Neudorf conceptualized the research question and analysis. Daniel Fuller, Joshua Neudorf, Stuart Lockhart, Charles Plante, Hazel Roberts and Thilina Bandara conducted the data analysis. Daniel Fuller, Stuart Lockhart and Hazel Roberts prepared the initial draft of the manuscript, and all of the authors commented on or reviewed draft versions of the manuscript. All of the authors reviewed the final version of the manuscript before submission, gave approval of the version to be published and agreed to be accountable for all aspects of the work.

Funding: Saskatchewan Health Research Foundation.

Supplemental information: For reviewer comments and the original submission of this manuscript, please see www.cmajopen.ca/content/7/1/ E33/suppl/DC1. 